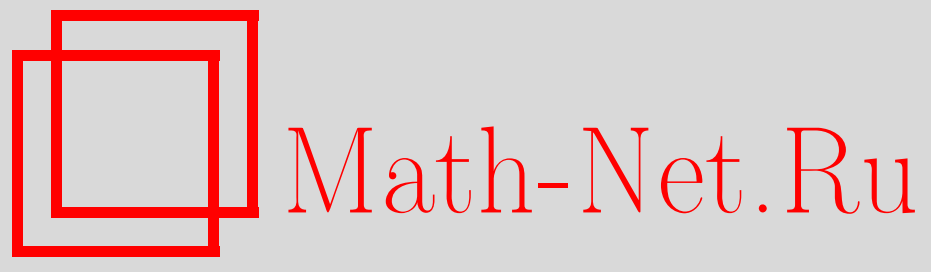

В. Н. Дубинин, О принципах мажорации для мероморфных функций, Матем. заметки, 2008, том 84, выпуск 6, 803-808

DOI: https://doi.org/10.4213/mzm6565

Использование Общероссийского математического портала Math-Net.Ru подразумевает, что вы прочитали и согласны с пользовательским соглашением http://www . mathnet.ru/rus/agreement

Параметры загрузки:

IP : 54.174 .149 .18

26 апреля 2023 г., 17:20:49

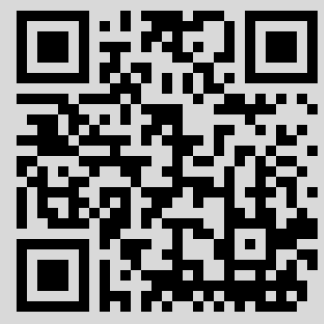


Том 84 выпуск 6 декабрь 2008

\section{О принципах мажорации для мероморфных функций}

\section{В. Н. Дубинин}

Рассматриваются дополнения к принципу Линделёфа о поведении функции Грина и принципу Неванлинны о поведении гармонической меры при мероморфных отображениях. Эти дополнения восходят к работам И. П. Митюка об изменении внутреннего радиуса области при отображении регулярными функциями.

Библиография: 11 названий.

Моему учителю Игорю Петровичу Митюку посвящается

1. Введение и формулировки результатов. Функцию Грина $g_{D}(z, \zeta)$ области $D$ называют классической, если она обращается в нуль на границе $D$. В этом случае доопределим функцию $g_{D}(z, \zeta)$ нулем в дополнении $\overline{\mathbb{C}}_{z} \backslash D$. Следующее утверждение принято называть принципом Линделёфа [1]. Пусть области $D$ и $G$ расширенной комплексной плоскости имеют классические функции Грина, и пусть функция $f: D \rightarrow G$ мероморфна в $D$. Тогда для любой фиксированной конечной точки $w_{0}$, принадлежащей $G$, справедливо неравенство

$$
g_{G}\left(f(z), w_{0}\right) \geqslant \sum_{k \geqslant 0} g_{D}\left(z, z_{k}\right), \quad z \in D,
$$

где $z_{0}, z_{1}, \ldots, z_{k}, \ldots$ - нули функции $f-w_{0}$, причем каждый из них учитывается столько раз, какова его кратность. Равенство в (1) в какой-либо точке $z \in D$ влечет за собой равенство в (1) во всей области $D$. Даже ослабленный вариант этого принципа

$$
g_{G}\left(f(z), w_{0}\right) \geqslant g_{D}\left(z, z_{0}\right)
$$

является существенным обобщением леммы Шварца и имеет многочисленные приложения в геометрической теории функций (см., например, [1], [2]). Распространение принципа Линделёфа на случай функций Робена вместо функций Грина дано в недавней работе [3]. Обозначим через $r(D, \zeta)$ внутренний радиус области $D$ относительно точки $\zeta \in D$, определяемый соотношением

$$
g_{D}(z, \zeta)=-\log |z-\zeta|+\log r(D, \zeta)+o(1), \quad z \rightarrow \zeta .
$$

Работа выполнена при поддержке Российского фонда фундаментальных исследований (грант № 08-01-00028) и ДВО РАН (грант № 06-III-A-01-013).

(C) В. Н. Дувинин, 2008 
В работе [4] Митюк заметил, что из неравенства (1) вытекает неравенство

$$
r\left(G, w_{0}\right) \geqslant\left|a_{p}\right| r^{p}\left(D, z_{0}\right) \exp \sum_{k \geqslant 1} g_{D}\left(z_{0}, z_{k}\right),
$$

где $f(z)=w_{0}+a_{p}\left(z-z_{0}\right)^{p}+\cdots$ - регулярная функция в области $D$. Более того, Митюк показал эффективное применение последнего неравенства в сочетании с методами симметризации [4]-[7]. В пособии [6] впервые указано на необходимость изучения обратных неравенств. Именно, следуя [6], рассмотрим функцию $f(z)=w_{0}+a_{p}\left(z-z_{0}\right)^{p}+\cdots$, регулярную в области $D$, и пусть $A-$ множество предельных точек последовательностей $\left\{f\left(z_{\nu}^{\prime}\right)\right\}$, когда $z_{\nu}^{\prime} \in D, z_{\nu}^{\prime} \rightarrow \partial D, \nu \rightarrow \infty$. Фиксируем конечную точку $w_{0} \notin A$ и обозначим через $G$ связную компоненту дополнения множества $A$, содержащую точку $w_{0}$. Тогда

$$
r\left(G, w_{0}\right) \leqslant\left|a_{p}\right| r^{p}\left(D, z_{0}\right) \exp \sum_{k \geqslant 1} g_{D}\left(z_{0}, z_{k}\right),
$$

причем равенство в (4) имеет место в том и только том случае, когда $G=f(D)$ и функция $f$ производит полное $n$-кратное накрытие области $G$ областью $D$ (здесь $n$ - суммарная кратность нулей функции $\left.f-w_{0}\right)$. Последнее означает, что при стремлении точки $z$ к границе области $D$ все предельные значения функции $f(z)$ принадлежат границе $G$ и каждой точке $w \in G$ соответствует ровно $n$ прообразов в области $D$ (с учетом кратности) [6; с. 22]. Сказанное выше можно перефразировать следующим образом: если $f(\partial D) \subset \overline{\mathbb{C}}_{w} \backslash G$, то справедливо неравенство (4). Заметим, что неравенство (3) выполняется при условии $f(D) \subset G$. Однако для неоднолистной функции $f$ противоположного включения $f(D) \supset G$ недостаточно для выполнения неравенства (4), как показывает пример функции $f(z)=z^{2}$ и областей $D=\mathbb{C}_{z} \backslash\{z=x+i y:-\infty \leqslant x \leqslant-1, y=0\}$ и $G=\{w:|w|<R\}$ при любом $R>16\left(z_{0}=w_{0}=0\right)$. Следующее утверждение содержит неравенство (4) как следствие при $z \rightarrow z_{0}$.

Теорема 1. Пусть области $D$ и $G$ имеют классические функции Грина, $D \subset$ $\overline{\mathbb{C}}_{z}, G \subset \overline{\mathbb{C}}_{w}$, и пусть $w_{0}-\oint$ фисированная точка области $G$. Предположим, что функция $f$ является мероморфной в области $D$, принимает по крайней мере один раз значение $w_{0}$ в $D$ и удовлетворяет условию $f(\partial D) \subset \overline{\mathbb{C}}_{w} \backslash G$ (т.е. при стремлении точки z $\kappa$ границе области $D$ все предельные значения функции $f(z)$ лежат в дополнении $G$ ). Тогда для любой точки $z \in D$ справедливо неравенство

$$
g_{G}\left(f(z), w_{0}\right) \leqslant \sum_{k=0}^{m} g_{D}\left(z, z_{k}\right),
$$

где $z_{0}, \ldots, z_{m}-$ нули функции $f-w_{0}$ в случае $w_{0} \neq \infty$ и нули функции $1 / f$ при $w_{0}=\infty$, причем каждый нуль учитывается столько раз, какова его кратность. Равенство в (5) в какой-нибудв точке $z \in D$ влечет за собой равенство в (5) во всей области $D$ и выполняется тогда и только тогда, когда $G=f(D)$ и функиия $f$ осуществляет полное $(m+1)$-кратное накрытие области $G$ областью $D$.

Неравенство (5) впервые встречается в работе Митюка [8] при несколько иных ограничениях на функцию $f$. Митюк рассматривал (5) как вспомогательное неравенство для иного, чем в [6], варианта доказательства оценки (4), дополняющей 
его ранний результат (3). Вместе с тем, теорема 1 является важным дополнением к принципу Линделёфа и, как выяснилось много лет спустя, она содержит, в частности, многие классические и современные неравенства для полиномов и рациональных функций [9]. Отметим, что из неравенства (5) вытекает следующее утверждение (ср. следствие 2 работы [9]).

СлЕДСтвиЕ 1. Пусть в условиях теоремы 1 область D имеет на своей гранище открытую аналитическую дугу Жордана $\gamma, \infty \notin \gamma$, ни одна точка которой не является предельной для граничных точек, не принадлежащих $\gamma$, а область $G$ имеет на своей гранище такую же дугу Г. Предположим, что функция $f$ отображает дугу $\gamma$ на некоторую дугу $f(\gamma) \subset \Gamma$ так, что положительной ориентации $\gamma$ соответствует положительная ориентация на ${ }^{1}{ }^{1}$ Тогда для любой точки $z \in \gamma$ справедливо неравенство

$$
\left|f^{\prime}(z)\right| \frac{\partial g_{G}\left(f(z), w_{0}\right)}{\partial n} \leqslant \sum_{k=0}^{m} \frac{\partial g_{D}\left(z, z_{k}\right)}{\partial n}
$$

где Ә/Әп означает дифферениирование вдоль внутренней нормали к соответствующей граничной дуге. Равенство в какой-либо точке $z \in \gamma$ достигается лишь в случае, указанном в теореме 1.

Интегрируя последнее соотношение, приходим к дополнению принципа мажорации Неванлинны о поведении гармонической меры [1]. Ранее такого рода дополнения рассматривались Лукашовым [10]. В данной заметке доказывается более общий, чем в [10], результат, причем с утверждением о знаке равенства. Обозначим через $\omega(z, \alpha, D)$ гармоническую меру множества $\alpha$ относительно области $D$ в точке $z[1]$, $[2]$.

Теорема 2. Пусть области $D \subset \overline{\mathbb{C}}_{z} u G \subset \overline{\mathbb{C}}_{w}$ ограничены конечным числом жордановых кривых, $w_{0}-$ точка области $G$. Пусть функция $f$ мероморфна в $D$, принимает по крайней мере один раз значение $w_{0}$ в $D$ и удовлетворяет условию $f(\partial D) \subset \overline{\mathbb{C}}_{w} \backslash G$. Предположим, что множества $\gamma \in \partial D$ u $f(\gamma) \subset \partial G$ состоят из конечного числа открытых дуг, причем положительной ориентации дуг из $\gamma$ соответствует при отображении $f$ положительная ориентация на $\partial G$. Тогда

$$
\omega\left(w_{0}, f(\gamma), G\right) \leqslant \sum_{k=0}^{m} \omega\left(z_{k}, \gamma, D\right),
$$

где $z_{0}, \ldots, z_{m}$ - нули функции $f-w_{0}$ в случае $w_{0} \neq \infty$ и нули функции $1 / f$ при $w_{0}=\infty$, причем каждый нуль учитывается столько раз, какова его кратность. Равенство в (7) имеет место в том и только том случае, когда функция $f$ осуществляет полное $(m+1)$-кратное накрытие области $G$ областью $D, G=f(D)$, и отображение $f: \gamma \rightarrow f(\gamma)$ является взаимно однозначным соответствием.

\footnotetext{
13десь дуги $\gamma$ и Г являются граничными кривыми, при обходе которых соответствующая область остается слева, при этом граничные точки считаются различными, если они являются носителями различных достижимых граничных точек, а предельная точка понимается в соответствующей топологии.
} 


\section{2. Доказательства.}

ДоКАЗАТЕЛЬСтво теОРЕМЫ 1. В условиях теоремы 1 функция $f$ принимает значение $w_{0} \in G$ лишь в конечном числе точек $z_{0}, z_{1}, \ldots, z_{m}$ области $D$. В противном случае существует последовательность точек $z_{\nu}^{\prime} \in D, z_{\nu}^{\prime} \rightarrow \partial D, \nu \rightarrow \infty$, такая, что $f\left(z_{\nu}^{\prime}\right)=w_{0}$, что противоречит условию $f(\partial D) \subset \overline{\mathbb{C}}_{w} \backslash G$. Привлекая дробно-линейные отображения, можно считать, что все точки $z_{0}, z_{1}, \ldots, z_{m}$ и $w_{0}$ конечные. Рассмотрим вспомогательную функцию

$$
h(z):=g_{G}\left(f(z), w_{0}\right)-\sum_{k=0}^{m} g_{D}\left(z, z_{k}\right), \quad z \in D .
$$

Так как функция $g_{G}\left(w, w_{0}\right)$ - субгармоническая в $\overline{\mathbb{C}}_{w} \backslash w_{0}$, то суперпозиция $g_{G}(f(z)$, $\left.w_{0}\right)$ - субгармоническая в области $D \backslash \bigcup_{k=0}^{m}\left\{z_{k}\right\}$ и, следовательно, функция $h(z)-$ также субгармоническая в этой области. Пусть теперь $z_{k}-$ нуль функции $f-w_{0}$ кратности $n_{k}, 0 \leqslant k \leqslant m$. В окрестности точки $z_{k}$ имеем

$$
\begin{gathered}
g_{G}\left(f(z), w_{0}\right)=-\log \left|f(z)-w_{0}\right|+h_{1}=-n_{k} \log \left|z-z_{k}\right|+h_{2}, \\
\sum_{k=0}^{m} g_{D}\left(z, z_{k}\right)=-n_{k} \log \left|z-z_{k}\right|+h_{3},
\end{gathered}
$$

где $h_{j}, j=1,2,3,-$ гармонические функции. Таким образом, особенности сокращаются и функция $h(z)$ субгармоническая во всей области $D$. Из условия теоремы для любой граничной точки $\zeta \in \partial D$

$$
\varlimsup_{z \rightarrow \zeta} h(z)=0 .
$$

По принципу максимума

$$
h(z) \leqslant 0 \quad \text { на } D,
$$

что равносильно неравенству (5), причем равенство в одной точке влечет за собой равенство во всей области $D$.

Покажем, что в случае равенства в (5) выполняется $G=f(D)$ и функция $f$ осуществляет полное $(m+1)$-кратное накрытие области $G$ областью $D$. Ввиду свойств аналитической функции и условия $f(\partial D) \subset \overline{\mathbb{C}}_{w} \backslash G$ каждая точка $w$ области $G$ имеет некоторую окрестность $U_{w}$, обладающую следующим свойством: число прообразов $f^{-1}\left(w^{\prime}\right)$ одно и то же для любой точки $w^{\prime} \in U_{w}$ (возможно, нулевое). Заметим, что точка $w_{0}$ обладает окрестностью, для которой это число равно $m+1$. Поскольку любую другую точку области $G$ можно соединить с $w_{0}$ кривой в $G$ и покрыть эту кривую конечным числом указанных окрестностей, каждой точке $G$ соответствует ровно $m+1$ прообразов. В частности, $f(D) \supset G$. Если теперь в (5) имеет место равенство для любой точки $z \in D$, то необходимо $f(D) \subset G$ и, следовательно, $f(D)=G$. Вновь учитывая условие $f(\partial D) \subset \overline{\mathbb{C}}_{w} \backslash G$, заключаем, что $f(\partial D) \subset \partial G$. Наоборот, пусть $G=f(D)$ и функция $f$ осуществляет полное $(m+1)$-кратное накрытие области $G$ областью $D$. Тогда функция $h$ - гармоническая в $D$ и на границе $D$ обращается в нуль. Следовательно, $h \equiv 0$ в $D$, что равносильно равенству в (5). Теорема 1 доказана. 
ДОКАЗАТЕЛЬСТво СЛЕДСТВИЯ 1 . В условиях следствия 1 функция $f$ продолжима на дугу $\gamma$ так, что производная $f^{\prime}(z)$ имеет смысл при $z \in \gamma$ (см., например, [6; гл. VI, п. 16]). Далее, в достаточно малой полуокрестности точки $z \in \gamma$ выполняется $h(z) \leqslant 0$, а на соответствующем участке $\gamma$ имеем $h(z)=0$. Следовательно, $\partial h / \partial n \leqslant 0$ в указанной точке. Учитывая сохранение положительной ориентации при отображении $f$, получаем неравенство (6). Если теперь в некоторой $z \in \gamma$ имеет место равенство в $(6)$, то, во-первых, $f^{\prime}(z) \neq 0$, так как в противном случае приходим к противоречию лемме Хопфа для слагаемых в правой части (6) [11]. При $f^{\prime}(z) \neq 0$ равенство в (6) равносильно равенству $\partial h / \partial n=0$, что вновь по лемме Хопфа дает $h \equiv 0$ в $D$. Отсюда выполняются условия равенства в теореме 1 . Следствие 1 доказано.

ДокАЗАТЕЛЬСтво тЕоремы 2. Так как гармоническая мера является конформным инвариантом, то при доказательстве теоремы 2 можно считать, что области $D$ и $G$ ограничены конечным числом аналитических жордановых кривых. Из неравенства (6) следует

$$
\begin{aligned}
\omega\left(w_{0}, f(\gamma), G\right) & =\frac{1}{2 \pi} \int_{f(\gamma)} \frac{\partial g_{G}\left(w, w_{0}\right)}{\partial n} d s \leqslant \frac{1}{2 \pi} \int_{\gamma} \frac{\partial g_{G}\left(f(z), w_{0}\right)}{\partial n}\left|f^{\prime}(z)\right| d s \\
& \leqslant \frac{1}{2 \pi} \int_{\gamma} \sum_{k=0}^{m} \frac{\partial g_{D}\left(z, z_{k}\right)}{\partial n} d s=\sum_{k=0}^{m} \omega\left(z_{k}, \gamma, D\right) .
\end{aligned}
$$

Итоговое неравенство совпадает с (7). Если теперь в (7) имеет место знак равенства, то равенство выполняется во всех промежуточных соотношениях. В частности, ввиду непрерывности подынтегральных функций имеем равенство в (6) и, следовательно, $G=f(D)$, а функция $f$ осуществляет полное $(m+1)$-кратное накрытие области $G$ областью $D$. Далее, если предположить, что две различные точки множества $\gamma$ переходят при отображении $f$ в одну точку на $f(\gamma)$, то существуют две открытые дуги на $\gamma$, переходящие в одну и ту же дугу на $f(\gamma)$, что при интегрировании положительной функции приводит к строгому неравенству в (7). Наоборот, пусть функция $f$ осуществляет полное $(m+1)$-кратное накрытие области $G$ областью $D, G=f(D)$, и отображение $f: \gamma \rightarrow f(\gamma)$ является взаимно однозначным соответствием. Тогда неравенство (6) и выписанные выше неравенства для интегралов превращаются в равенства. Это завершает доказательство теоремы 2.

\section{СПИСОК ЦИТИРОВАННОЙ ЛИТЕРАТУРЫ}

[1] С. Стоилов, Теория функиий комплексного переменного, т. 2, ИЛ, М., 1962.

[2] Г. М. Голузин, Геметрическая теория функиий комплексного переменного, Наука, М., 1966.

[3] V. N. Dubinin, M. Vuorinen, "Robin functions and distortion theorems for regular mappings", Preprint 454, Reports in Mathematics, Univ. of Helsinki, Helsinki, 2007.

[4] И.П. Митюк, "Принцип симметризации для многосвязных областей", Докл. АН CCCP, 157:2 (1964), 268-270.

[5] И. П. Митюк, "Принцип симметризации для многосвязных областей и некоторые его применения", Укр. матем. журн., 17:4 (1965), 46-54.

[6] И. П. Митюк, Симметризационные методы и их применение в геометрической теории функиий. Введение в симметризационные методы, Кубанский гос. ун-т, Краснодар, 1980. 
[7] И. П. Митюк, Применение симметризационных методов в геометрической теории функций, Кубанский гос. ун-т, Краснодар, 1985.

[8] И.П. Митюк, "Оценки внутреннего радиуса (емкости) некоторой области (конденсатора)", Изв. Северо-Кавказ. научн. чентра высш. шк. естеств. наук., 1983, №3, $36-38$.

[9] В.Н. Дубинин, С. И. Калмыков, "Принцип мажорации для мероморфных функций", Матем. сб., 198:12 (2007), 37-46.

[10] А. Л. Лукашов, “Дополнения к принципу гармонической меры Р. Неванлинны”, Maтем. заметки, 84:4 (2008), 632-633.

[11] M. H. Protter, H.F. Weinberger, Maximum Principles in Differential Equations, Springer-Verlag, New York, 1984.

\section{В. Н. Дубинин}

Поступило

Институт прикладной математики ДВО РАН

14.03.2008

E-mail: dubinin@iam.dvo.ru 